\title{
Diode-Laser Induced Fluorescence Spectroscopy of an Optically Thick Plasma in Combination with Laser Absorption Spectroscopy
}

\author{
S. Nomura, ${ }^{1}$ T. Kaneko, ${ }^{2}$ G. Ito, ${ }^{1}$ K. Komurasaki, ${ }^{1}$ and Y. Arakawa ${ }^{2}$ \\ ${ }^{1}$ Department of Advanced Energy, The University of Tokyo, Tokyo 277-0882, Japan \\ ${ }^{2}$ Department of Aerospace Engineering, The University of Tokyo, Tokyo 113-8656, Japan \\ Correspondence should be addressed to S. Nomura; nomura@al.t.u-tokyo.ac.jp
}

Received 13 November 2012; Revised 11 January 2013; Accepted 21 January 2013

Academic Editor: Lorenzo Giuffrida

Copyright (c) 2013 S. Nomura et al. This is an open access article distributed under the Creative Commons Attribution License, which permits unrestricted use, distribution, and reproduction in any medium, provided the original work is properly cited.

\begin{abstract}
Distortion of laser-induced fluorescence profiles attributable to optical absorption and saturation broadening was corrected in combination with laser absorption spectroscopy in argon plasma flow. At high probe-laser intensity, saturated absorption profiles were measured to correct probe-laser absorption. At low laser intensity, nonsaturated absorption profiles were measured to correct fluorescence reabsorption. Saturation broadening at the measurement point was corrected using a ratio of saturated to non-saturated broadening. Observed LIF broadening and corresponding translational temperature without correction were, respectively, $2.20 \pm 0.05 \mathrm{GHz}$ and $2510 \pm 100 \mathrm{~K}$ and corrected broadening and temperature were, respectively, $1.96 \pm 0.07 \mathrm{GHz}$ and $1990 \pm 150 \mathrm{~K}$. Although this correction is applicable only at the center of symmetry, the deduced temperature agreed well with that obtained by LAS with Abel inversion.
\end{abstract}

\section{Introduction}

Diode laser-induced fluorescence (DLIF) has a feature of high wavelength resolution on the order of picometers, which makes it useful to obtain translational temperature by measuring Doppler broadening of an atomic line of gases [ 1 , 2 ] to the same degree as diode laser absorption spectroscopy (DLAS) $[3,4]$. The advantage of DLIF over DLAS, which is a line-of-sight measurement, is the possibility of point measurements. However, in optically thick plasma, absorption of the excitation laser and reabsorption (or self-absorption) of fluorescence can broaden the fluorescence profile. The temperature deduced from observed fluorescence tends to be overestimated. Hertz-corrected DLIF profiles in flame consider the laser absorption effect by solving a nonsymmetric 1D distribution of the depleting laser using an iterative procedure [5], although fluorescence reabsorption was not corrected.

Although intense lasers with substantial fluorescence that can achieve a high signal-to-noise ratio are preferred, intense lasers are known to cause additional broadening, termed as saturation broadening or power broadening [6]. Moreover, their saturation regime is usually avoided. Instead, the linear region is used in LIF temperature measurements [7]. In contrast, in low-pressure plasma, the saturation intensity is low and the fluorescence profile is easily saturated. The saturation effect in LIF was investigated in earlier studies [810], in which the laser spectral width was comparable to Doppler broadening, and in which the spectral wing of laser was able to induce substantial fluorescence, causing additional broadening. In DLIF, however, the laser spectral width is about $1 \mathrm{MHz}$ and three others of magnitude narrower than the absorption profile broadening. The mechanism of broadening differs, but with identical broadening to that of LAS [1113].

Correction methods for optical absorption and saturation broadening are demonstrated in an arc-heated argon plasma wind tunnel [14]. The wavelengths of both fluorescence and excitation are $772.42 \mathrm{~nm}\left(\operatorname{Ar~I}, 4 \mathrm{~s}^{2}[1 / 2]^{\circ}-4 \mathrm{p}^{2}[1 / 2]\right)$. The measured point is on the axis of the axisymmetric flow and the measured temperature is compared with the result of LAS with Abel inversion. 


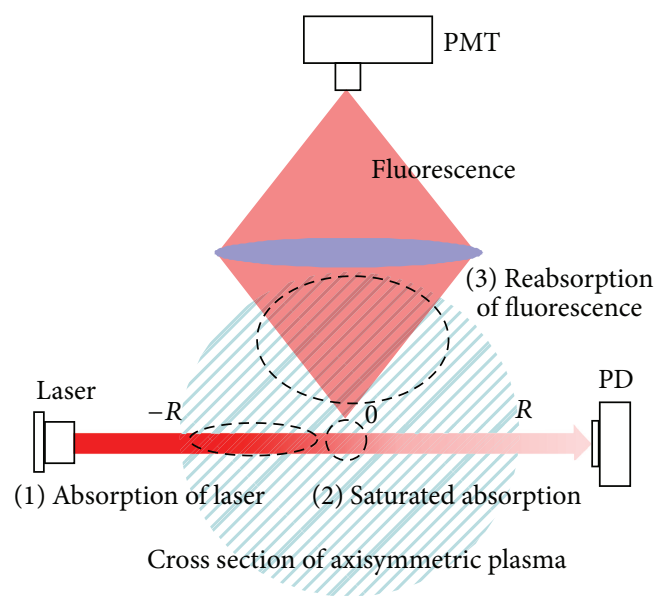

FIGURE 1: Schematic of LIF combined with LAS.

\section{Theoretical}

2.1. Absorption of the Exciting Laser. Taking laser absorption into consideration, the fluorescence is written as [5]

$$
F_{0}=C k_{s}(0) I_{\text {in }} \exp \left(-\int_{-R}^{0} k_{s}(x) d x\right),
$$

where $F_{0}, C, I_{\text {in }}$, and $k_{s}$, respectively, denote the fluorescence intensity without reabsorption, experimental factor, incident spectral laser irradiance, and saturated absorption coefficient. The coordinate system is presented in Figure 1 . The $x$-axis is the laser pass and the center of the flow is the origin point. $k_{s}(0)$ is the saturated absorption coefficient at $x=0$. It is noteworthy that the laser absorption is saturated because of its high irradiance. In the axisymmetric plasma, absorbance from the incident edge to the center is half of that from the incident edge to the edge, as

$$
\int_{-R}^{0} k_{s}(v, x) d x=\frac{1}{2} \int_{-R}^{R} k_{s}(v, x) d x,
$$

where $v$ is the laser frequency. The factor of $1 / 2$ in (2) is valid only for the axisymmetric plasma. In accordance with the Beer-Lambert law, integration of the right term in (2) is

$$
\int_{-R}^{R} k_{s}(\nu, x) d x=-\ln \left(\frac{I}{I_{\text {in }}}\right),
$$

where $I$, respectively, denote the transmitted spectral laser irradiance. Consequently the laser absorption can be corrected by monitoring the transmitted spectral laser irradiance.

2.2. Reabsorption of the Fluorescence. The fluorescence intensity is much lower than the saturation intensity, and the absorption of fluorescence is not saturated. Consequently correction for the reabsorption must be done with the nonsaturated absorption, which is measured at low intensity. The measured fluorescence is expressed as

$$
F=F_{0} \exp \left(-\int_{0}^{R} k(x) d x\right),
$$

where $F$ and $k$, respectively, denote the measured fluorescence and nonsaturated absorption coefficient. In the same manner as the correction for the absorption of laser, the absorption can be evaluated:

$$
\int_{0}^{R} k(x) d x=-\frac{1}{2} \ln \left(\frac{I}{I_{\mathrm{in}}}\right) .
$$

Although fluorescence may diffuse before it reaches the detector, this effect does not alter the profile shape because the diffusion is not a function of laser frequency in this spectral range. Thus diffusion is not considered here.

2.3. Saturation Effect. When intense laser illumination is used for excitation, the optical pumping rate exceeds the relaxation rate and the number density of the lower state atoms is depleted. The saturated integrated absorption coefficient is

$$
K_{s}=K \frac{1}{\sqrt{1+I / I_{s}}},
$$

where $K_{s}, K$, and $I_{s}$, respectively signify the saturated, nonsaturated integrated absorption coefficient, and saturation intensity. $K$ is integration over the frequency and has a unit of $\mathrm{GHz} / \mathrm{mm}$. A series of $K_{s}$ is obtained using LAS by varying the laser power. By fitting the measured $K_{s}$ with (6), $K$ and $I_{s}$ are obtainable. According to an earlier report [11], the true Doppler broadening is obtainable when the saturation parameter, $S\left(=I / I_{s}\right)$, is less than 0.1 . With a ratio of saturated broadening to the true Doppler broadening, the saturation effect is corrected.

\section{Experimental}

Figure 2 shows the experimental setup. An external cavity diode laser (Velocity Model 6300; New Focus Inc.), with linewidth of about $300 \mathrm{kHz}$, was used as the light source. The laser wavelength was swept at the repetitive frequency of $0.5 \mathrm{~Hz}$ with sweeping width of $20 \mathrm{GHz}$. The relative laser wavelength was monitored using an etalon with free spectral range of $0.75 \mathrm{GHz}$. The laser was modulated using an acoustooptic modulator (1205C; Isomet Corp.). The laser power at the output of the fiber was $1.0 \mathrm{~mW}$ and the laser spatial width at the measurement point was $0.4 \mathrm{~mm}$, at which the intensity decreases to $1 / e^{2}$ of the peak. During saturation measurements, the laser power was attenuated with neutral density filters. The fluorescence was collected with lenses and detected with a photomultiplier tube (H8249; Hamamatsu Photonics K.K.). A band-pass filter was mounted in front of the detector and the fluorescence signal was measured by phase sensitive detection using a lock-in amplifier (SR830; Stanford Research Systems Inc.). The plasma emission is negligibly small in comparison to the fluorescence signal. The output from the lock-in amplifier was recorded with an oscilloscope (DSOX2004A; Agilent Technologies Inc.).

The argon plasma flow was generated with an arc-heated plasma generator and expanded with a nozzle. The throat diameter was $2 \mathrm{~mm}$. The nozzle exit was $30 \mathrm{~mm}$. The input power was $1.2 \mathrm{~kW}$ with current of $50 \mathrm{~A}$. The volume flow rate of argon was $4 \mathrm{slm}$. The chamber pressure was $20 \mathrm{~Pa}$. 


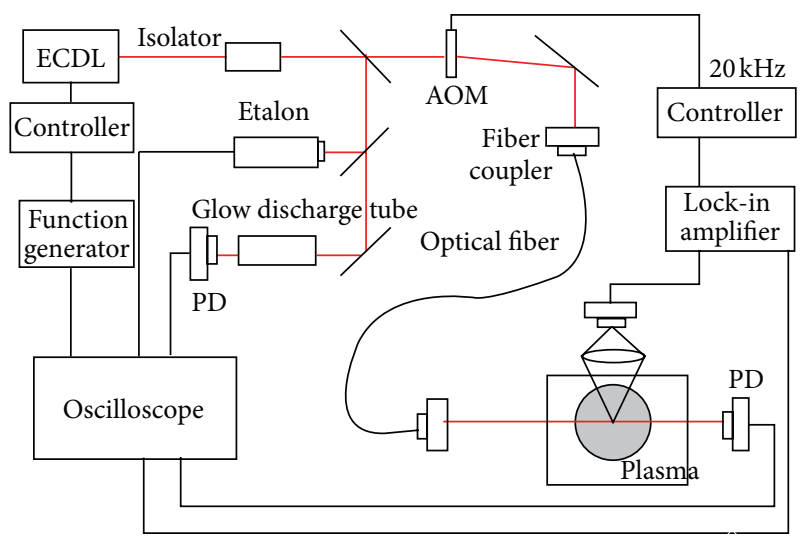

FIGURE 2: Schematic of LIF combined with LAS.

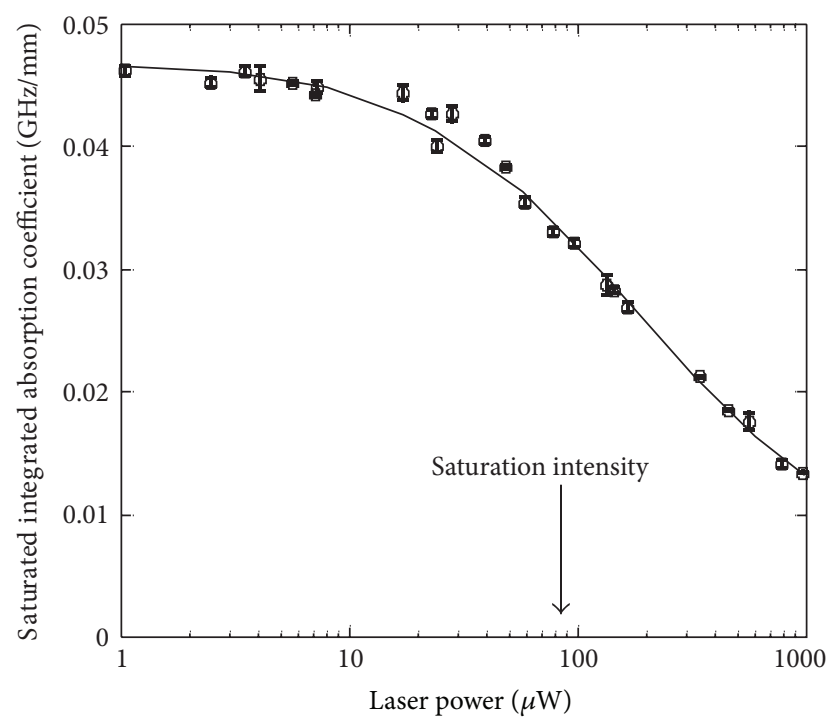

FigURE 3: Saturated integrated absorption coefficient as a function of laser power.

\section{Results and Discussion}

Saturated LAS was conducted with varying laser power from $1 \mu \mathrm{W}$ to $0.97 \mathrm{~mW}$. Figure 3 shows the saturated integrated absorption coefficient with the fitting curve. The saturation intensity was estimated at $86 \pm 4 \mu \mathrm{W}$. The nonsaturated absorbance was $0.403 \pm 0.006$ at the peak and the nonsaturated broadening was $1.80 \pm 0.02 \mathrm{GHz}$. The broadening was evaluated by using full width at half maximum (FWHM). The most saturated absorption profile was obtained with laser power of $0.97 \mathrm{~mW}$. At this power the saturated absorbance is $0.110 \pm 0.002$ at the peak; saturated broadening was $1.94 \pm$ $0.01 \mathrm{GHz}$. Consequently, the ratio of saturated broadening to true broadening was $1.08 \pm 0.02$.

The LIF profile was obtained with maximum laser power of $0.97 \mathrm{~mW}$. Broadening of the observed fluorescence was $2.20 \pm 0.05 \mathrm{GHz}$. The broadening effect by optical absorption was corrected using (1) and (4). Figure 4 shows the

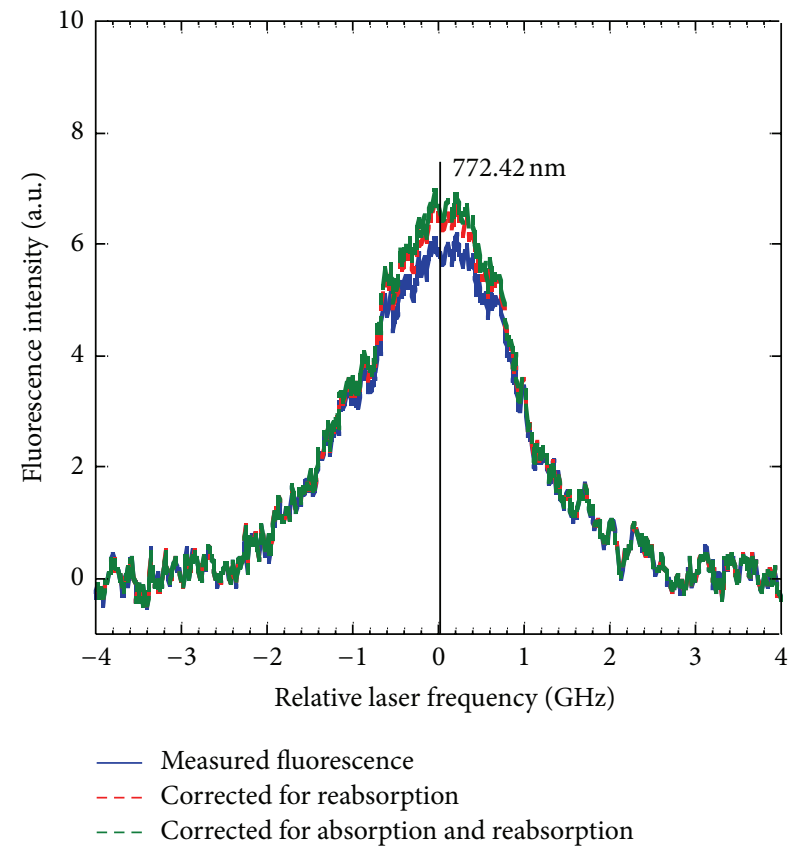

FIGURE 4: Fluorescence profiles of argon.

TABLE 1: Full width at half maximum (FWHM) and temperature of distorted profiles.

\begin{tabular}{lccc}
\hline & $\begin{array}{c}\text { FWHM, Temperature, } \\
\text { GHz }\end{array}$ & $\begin{array}{c}\text { Ratio of estimated } \\
\text { temperature to true } \\
\text { temperature }\end{array}$ \\
\hline $\begin{array}{l}\text { Profile distorted by } \\
\text { reabsorption }\end{array}$ & 2.04 & 2150 & 1.08 \\
$\begin{array}{l}\text { Profile distorted by } \\
\text { absorption }\end{array}$ & 1.98 & 2030 & 1.02 \\
$\begin{array}{l}\text { Profile distorted by } \\
\text { saturation }\end{array}$ & 2.11 & 2310 & 1.16 \\
\hline $\begin{array}{l}\text { Observed profile } \\
\text { True profile }\end{array}$ & 2.20 & 2510 & 1.26 \\
\hline
\end{tabular}

measured fluorescence profile and the corrected profiles. After correction for optical reabsorption, the broadening was deduced as $2.11 \pm 0.04 \mathrm{GHz}$. As described above, the ratio of saturated broadening to the true broadening is $1.08 \pm$ 0.02. Consequently, Doppler broadening and translational temperature were, respectively, deduced as $1.96 \pm 0.07 \mathrm{GHz}$ and $1990 \pm 140 \mathrm{~K}$. Broadening and temperature of distorted and true profiles are shown in Table 1.

Figure 5 presents the ratio of estimated temperature to true temperature as a function of $S$. The contribution of three effects to the distortion is shown there. The peak absorbance was 0.4 , which corresponds to the optical thickness-toplasma diameter ratio of $0.007 \mathrm{~mm}^{-1}$. The reabsorption of fluorescence was not saturated. It was independent of the spectral laser irradiance. As the spectral laser irradiance increased, the absorption of the laser became saturated and the distortion effect became minor. In the region where $S$ is higher than unity, saturation broadening is the most 


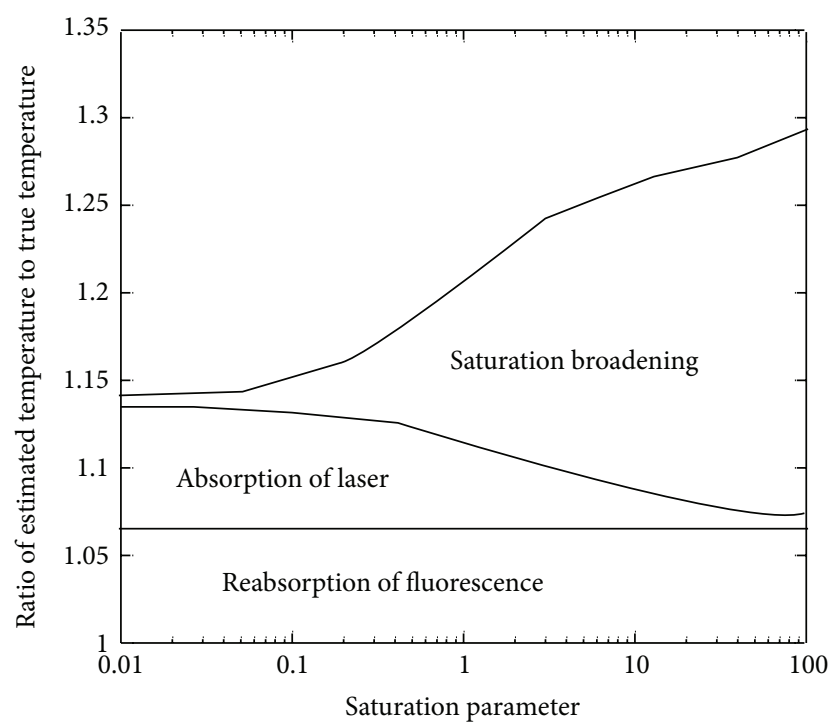

FIGURE 5: Ratio of LIF temperature (without correction) to true temperature as a function of saturation parameter, $I / I_{s}$. Optical thickness-to-plasma diameter ratio is $0.007 \mathrm{~mm}^{-1}$.

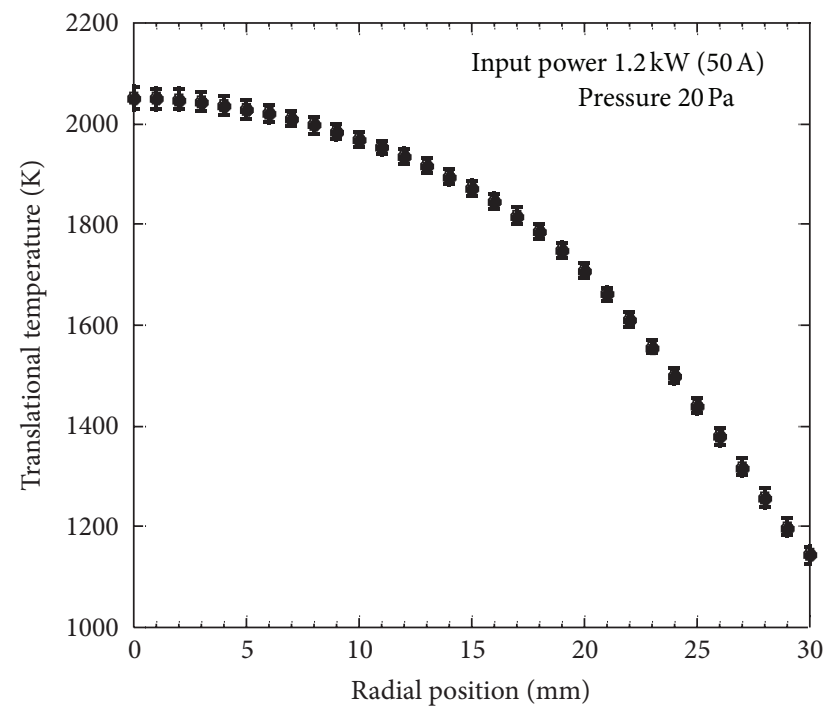

Figure 6: Temperature distribution obtained by laser absorption spectroscopy with Abel inversion.

dominant among distortion effects. The three effects are independent of each other, and the true temperature is obtainable by dividing the measured temperature by the ratio in Figure 5. Thus, the error propagates linearly.

For the validation of this correction method, the temperature distribution was obtained using LAS with Abel inversion, as shown in Figure 6. The laser power was attenuated to $2.5 \mu \mathrm{W}$ to avoid saturation. At the center, the temperature was estimated at $2050 \pm 20 \mathrm{~K}$. The present result shows good agreement with that obtained by LAS as shown in Table 2 .
TABLE 2: Translational temperature measured using LIF and LAS.

\begin{tabular}{ll}
\hline LIF & $1990 \pm 150 \mathrm{~K}$ \\
LAS & $2050 \pm 20 \mathrm{~K}$ \\
\hline
\end{tabular}

\section{Conclusion}

The distorted LIF profile attributable to optical absorption and saturation was corrected using LAS. For laser absorption and fluorescence reabsorption correction, saturated and nonsaturated line-of-sight absorbance was measured using the LAS setup. Saturation broadening was corrected with the ratio of saturated broadening to the nonsaturated broadening in LAS. The results obtained for arc-heated argon plasma show that the Doppler broadening was corrected as $1.96 \pm$ $0.07 \mathrm{GHz}$, and the translational temperature was $1990 \pm 150 \mathrm{~K}$. Instead, broadening without correction was $2.20 \pm 0.05 \mathrm{GHz}$, which corresponds to $2510 \pm 100 \mathrm{~K}$. This value showed good agreement with the result of LAS, which was spatially resolved with Abel inversion. The contribution of the three effects to distorted LIF profile was estimated theoretically as a function of $S$, showing that in the region where $S$ is higher than unity, saturation broadening is the most dominant. Although the present correction method is applicable only to the center measurement of axisymmetric plasma, this method is a powerful tool for the measurements of the temperature distribution along the flow axis.

\section{Conflict of Interests}

The authors do not have any conflict of interests.

\section{Acknowledgment}

This work was partly supported by Research Fellowships of the Japan Society for the Promotion of Science for Young Scientists.

\section{References}

[1] W. A. Hargus and C. S. Charles, "Near-plume laser-induced fluorescence velocity measurements of a medium power Hall thruster," Journal of Propulsion and Power, vol. 26, no. 1, pp. 135141, 2010.

[2] G. Galbács, Z. Galbács, O. Axner, and Z. Geretovszky, "Assessment and application of diode laser induced fluorescence spectrometry in an inductively coupled plasma to the determination of lithium," Spectrochimica Acta B, vol. 60, no. 3, pp. 299-306, 2005.

[3] S. Nomura, K. Komurasaki, S. Loehle, and G. Herdrich, "Evaluation of nonequilibrium excitation of inductively heated atomic Oxygen by laser absorption spectroscopy," Frontier of Applied Plasma Technology, vol. 4, no. 2, pp. 65-69, 2011.

[4] M. Matsui, H. Takayanagi, Y. Oda, K. Komurasaki, and Y. Arakawa, "Performance of arcjet-type atomic-oxygen generator by laser absorption spectroscopy and CFD analysis," Vacuum, vol. 73, no. 3-4, pp. 341-346, 2004.

[5] H. M. Hertz and M. Aldén, "Calibration of imaging laser-induced fluorescence measurements in highly absorbing flames," 
Applied Physics B Photophysics and Laser Chemistry, vol. 42, no. 2, pp. 97-102, 1987.

[6] W. Demtroeder, Laser Spectroscopy, Springer, 3rd edition, 2002.

[7] J. G. Liebeskind, R. K. Hanson, and M. A. Cappelli, "Laserinduced fluorescence diagnostic for temperature and velocity measurements in a hydrogen arcjet plume," Applied Optics, vol. 32, no. 30, pp. 6117-6127, 1993.

[8] M. J. Goeckner, J. Goree, and T. E. Sheridan, "Saturation broadening of laser-induced fluorescence from plasma ions," Review of Scientific Instruments, vol. 64, no. 4, pp. 996-1000, 1993.

[9] Y. Takubo, H. Yano, H. Matsuoka, and M. Shimazu, "Saturation behavior of laser-induced $\mathrm{CH}$ fluorescence in a propaneair flame," Journal of Quantitative Spectroscopy and Radiative Transfer, vol. 30, no. 2, pp. 163-168, 1983.

[10] N. Omenetto, P. Benetti, L. P. Hart, J. D. Winefordner, and C. T. J. Alkemade, "Non-linear optical behavior in atomic fluorescence flame spectrometry," Spectrochimica Acta B, vol. 28, no. 8, pp. 289-300, 1973.

[11] M. Matsui, K. Komurasaki, S. Ogawa, and Y. Arakawa, "Influence of laser intensity on absorption line broadening in laser absorption spectroscopy," Journal of Applied Physics, vol. 100, no. 6, Article ID 063102, 2006.

[12] S. Fornaca and M. Wickham, "Optical determination of velocity distribution," Bulletin of the American Physical Society, vol. 25, p. $991,1980$.

[13] D. N. Hill, S. Fornaca, and M. G. Wickham, "Single frequency scanning laser as a plasma diagnostic," Review of Scientific Instruments, vol. 54, no. 3, pp. 309-314, 1983.

[14] M. Matsui, T. Ikemoto, H. Takayanagi, K. Komurasaki, and Y. Arakawa, "Generation of highly dissociated oxygen flows using a constrictor-type arc heater," Journal of Thermophysics and Heat Transfer, vol. 21, no. 1, pp. 247-249, 2007. 

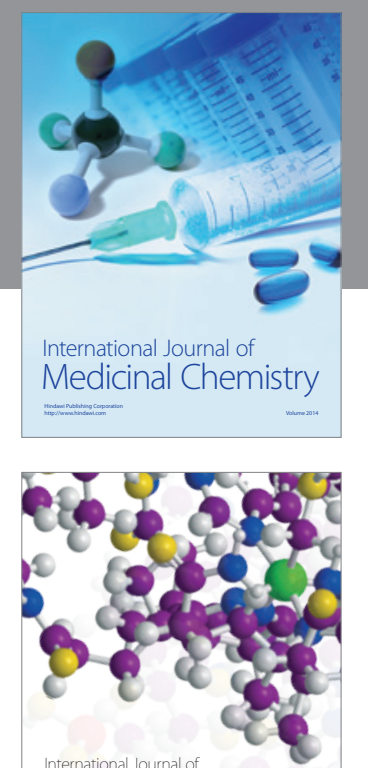

\section{Carbohydrate} Chemistry

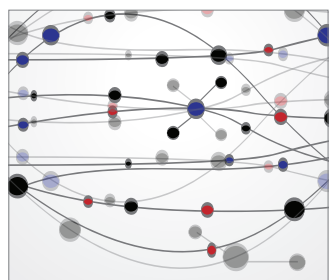

The Scientific World Journal
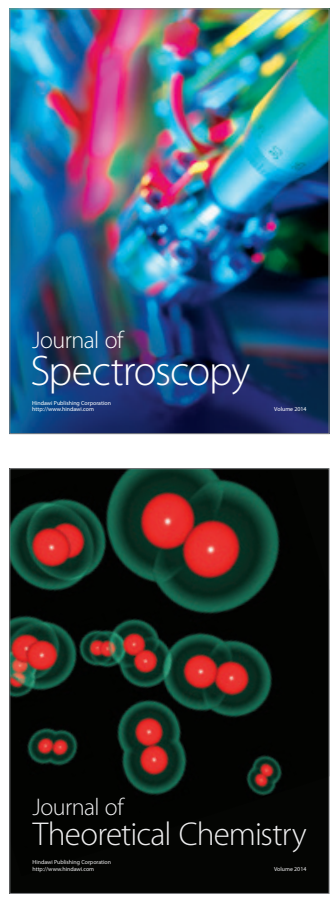
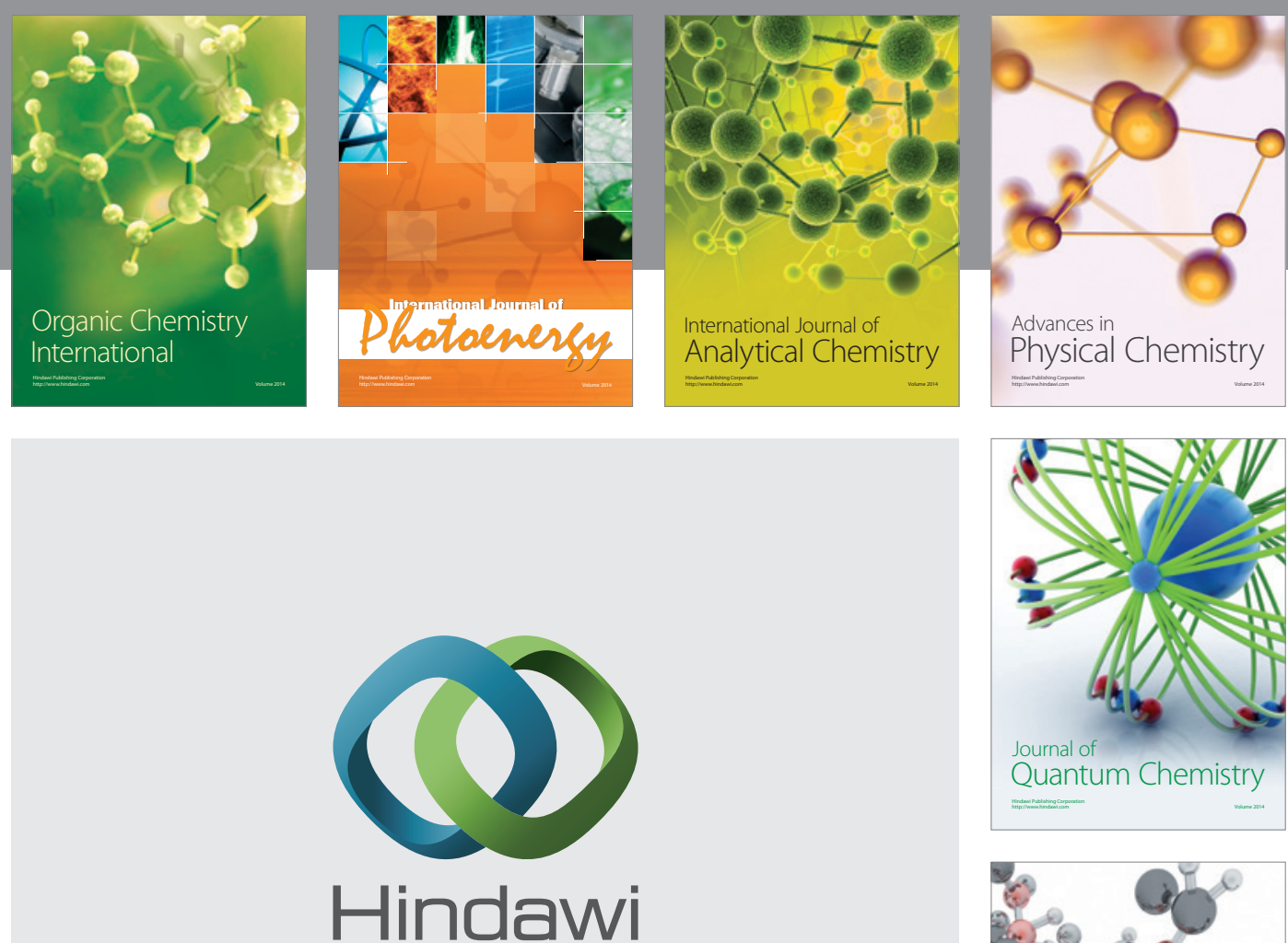

Submit your manuscripts at

http://www.hindawi.com

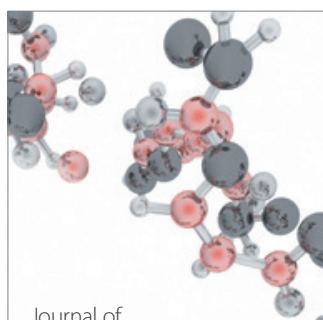

Analytical Methods

in Chemistry

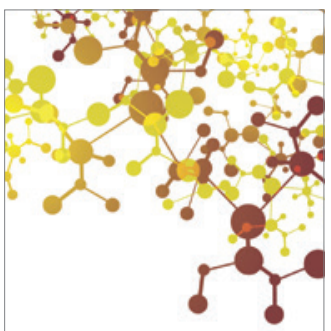

Journal of

Applied Chemistry

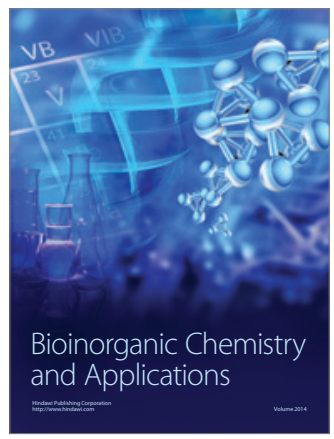

Inorganic Chemistry
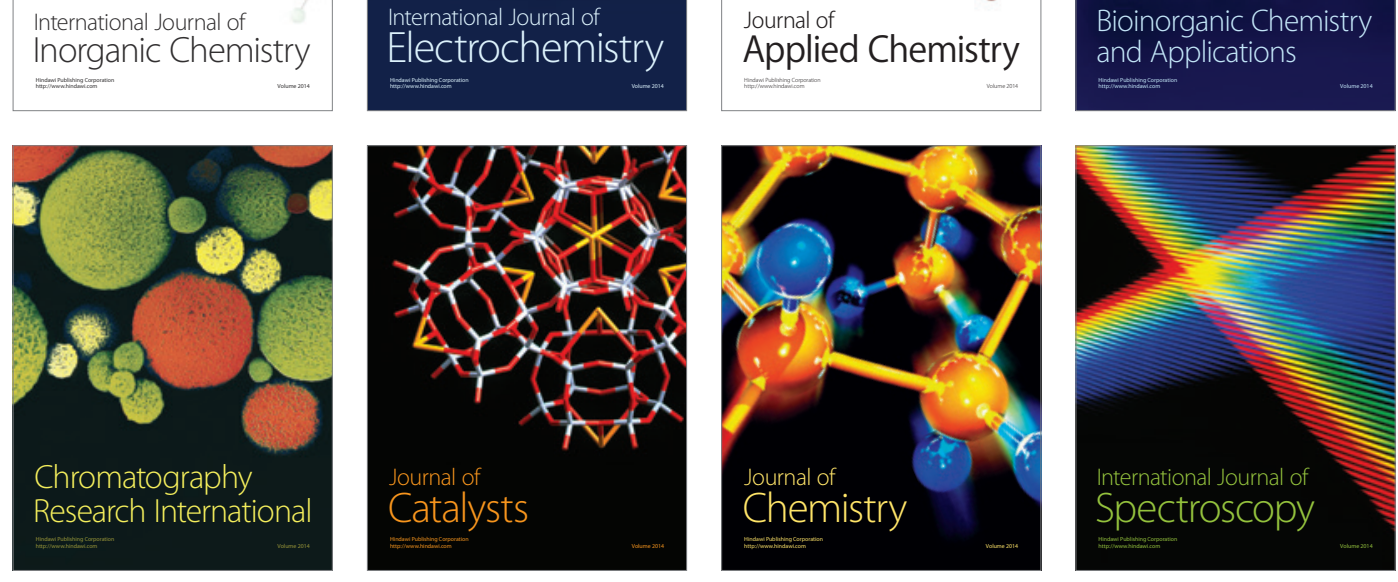